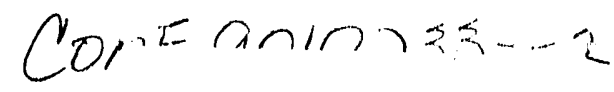

\title{
ELECTROMAGNETIC PRODUCTION OF STRANGE PARTICLES
}

\author{
Reinhard A. Schumacher
Department of Physics, Carnegie Mellon University, Pittsburgh, PA 15213
}

\section{litii}

\section{ABSTRACT}

A brief overview of the experimental status of electro- and photo-production of hyperons from a proton target is given; with some discussion of likely directions for future work.

\section{INTRODUCTION}

Electro- and photo-production of kaons and hyperons will soon enter a era of renewed experimental investigation with the advent of new facilities at new, high dutyfactor electron beam accelerators, such as CEBAF. We will discuss measurements of elementary strangeness electro-production, $\mathrm{p}\left(\mathrm{e}, \mathrm{e}^{\prime} \mathrm{K}\right) \mathrm{Y}$, and real photoproduction, $\mathrm{p}(\gamma, \mathrm{K}) \mathrm{Y}$. The physics motivation is focused on the production of strange quarks via the well-understood electromagnetic interaction. In contrast to hadronically induced reactions such as $(\pi, \mathrm{K})$ or $(\mathrm{p}, \mathrm{K})$, the photon is a relatively weakly interacting probe, allowing sensible first-order calculations to be done. These studies typically aim to extract values for the fundamental KYN coupling constants and to understand the dynamics of the strangeness-producing interaction. Good understanding of the elemertary interaction is a necessary step towards embedding it in the nuclear medium for the calculation of electromagnetic hypernuclear production.

While this talk will outline our knowledge of these reactions, it must be said ar the outset that many open questions remain in this field because the data are rather sparse. Also, present models typically evaluate Lorentz invariant amplitudes in terms of Feynman diagrams with exchange of mesons and baryons; contact with quark models is only indirect, through the calculation of coupling constants. The information in this talk is based on experiments done typically 15 to 25 years ago, some theoretical work done in recent years, and a discussion of what kinds of new results may be expected in the future.

Three related strangeness producing reactions on a proton target which lead to hyperons in the $\mathrm{J}^{\pi}=1 / 2^{+}$baryon octet can be considered:

$$
\begin{aligned}
\gamma_{(\mathrm{v})}+\mathrm{p} & \rightarrow \mathrm{K}^{+}+\Lambda & \left(\mathrm{E}_{\gamma_{\mathrm{Th}}}^{\mathrm{Th}}=0.911 \mathrm{GeV}\right) \\
& \rightarrow \mathrm{K}^{+}+\Sigma^{\mathrm{o}} & \left(\mathrm{E}_{\gamma}=1.046 \mathrm{GeV}\right) \\
& \rightarrow \mathrm{K}^{0}+\Sigma^{+} & \left(\mathrm{E}_{\gamma}^{\mathrm{Th}}=1.048 \mathrm{GeV}\right)
\end{aligned}
$$

where $E_{\gamma}^{T h}$ are the real photon threshold energies. The first two have been studied via both real and virtual photon experiments, while the third one has not yet been measured. Analogous reactions on the neutron and the production of excited hyperons have not been extensively studied.

In each of these reactions the final state hyperon can be polarized. Using the parity violating weak decay of these particles, this polarization can be measured withoy 
the need to do double scattering experiments. In unraveling the production mechanism this is an attractive feature in comparison to pion electro- and photo-production. For example, in the rest frame of the $\Lambda$, the weak decay $\Lambda \rightarrow \pi^{-} p$ emits the nucleon preferentially along the direction of the $\Lambda$ spin. For $\Lambda$ 's with polarization $P$, the decay yields a distribution $\mathrm{I}\left(\theta_{\mathrm{p}}\right)=\mathrm{I}_{0}\left(1+\alpha \mathrm{P} \cos \theta_{\mathrm{p}}\right)$, where the weak decay asymmetry parameter is $\alpha=0.642$, and $\theta_{p}$ is the angle between the nucleon momentum and the normal to the $\mathrm{K} \Lambda$ plane. For the $\Sigma^{+} \rightarrow \mathrm{p} \pi^{0}$ decay the weak asymmetry parameter is -0.98 . Because the $\Sigma^{\circ}$ decays $100 \%$ via an M1 electromagnetic transition to the $\Lambda$, a measurement of the decay $\Lambda$ polarization in Reaction 2 also measures the polarization of the $\Sigma^{0}$; the relationship is that $P_{\Lambda}=-1 / 3 P_{\Sigma}$.

\section{ELECTROPRODUCTION EXPERMMENTS}

In electroproduction experiments the relevant quantities are the 4-momentum of the virtual photon $\mathrm{q}=(\overrightarrow{\mathrm{q}}, \omega)$ and the direction $\left(\theta_{x}, \phi\right)$ of the hyperon relative to $\overrightarrow{\mathrm{q}}$ and the electron scattering plane, as shown in Figure 1. In terms of longitudinal and transverse photon polarization, the cross section can be written down most generally in terms of nine structure functions 1 ; this corresponds to knowledge of the electron's initial helicity state, the proton polarization, and the polarization of the produced hyperon. Some of the most ambitious experiments undertaken to date ${ }^{2], 3]}$ used unpolarized electrons on unpolarized protons, and detected the electrons and produced kaons but not the polarization of the hyperon. When initial state helicities, proton polarization, and hyperon polarization are averaged or summed over, only four of these nine structure functions are left. The experimental cross section takes the form 2],3],4]

$$
\frac{d^{4} \sigma}{d W d q^{2} d t d \phi}=\Gamma\left(\frac{d \sigma_{U}}{d t}+\varepsilon \frac{d \sigma_{L}}{d t}+\varepsilon \frac{d \sigma_{P}}{d t} \cos (2 \phi)+\sqrt{2 \varepsilon(\varepsilon+1)} \frac{d \sigma_{I}}{d t} \cos \phi\right)
$$

where the virtual photon flux is

$$
\Gamma=\frac{\alpha}{4 \pi^{2}} \frac{E_{e}}{E_{e}} \frac{W^{2}-M^{2}}{M Q^{2}} \frac{1}{1-\varepsilon}
$$

and the virtual photon polarization parameter is

$$
\varepsilon=\left(1+2 \frac{\overrightarrow{\mathrm{q}}^{2}}{\mathrm{Q}^{2}} \tan ^{2} \frac{\theta_{\mathrm{e}}}{2}\right)^{-1}
$$

Here $W$ is the total center of mass energy, $t$ the square of the 4-momentum transfer to the hyperon, $Q^{2}=-q^{2}$ the electron 4-momentum transfer, $M$ the proton mass, and $E_{e}$, $E_{e}{ }^{\prime}$, and $\theta_{e}$ are the electron energies and angle in the lab frame. The first two terms are the (e,e') inclusive (unpolarized) transverse and longitudinal photon cross sections, separable by the Rosenbluth method or by $180^{\circ}$ scattering. When the direction of the kaon is detected at the azimuthal angle $\phi$ out of the $\left(e, e^{\prime}\right)$ scattering plane, the transverse photons can be polarized parallel or perpendicular to the hyperon production plane. The term $\varepsilon d \sigma_{\mathrm{p}} / \mathrm{dt} \cos (2 \phi)$ is due to the interference of transverse components of this polarization. The term $(2 \varepsilon(\varepsilon+1))^{1 / 2} \mathrm{~d} \sigma_{1} / \mathrm{dt} \cos \phi$ is due to the interference between the 
transverse part of the transverse polarization and the longitudinal photon polarizations. The cross sections are functions of $W, q^{2}$, and $t$.

In the one-photon exchange shown in Figure 1 the interaction depends on the product of a leptonic current, $J_{\mu}(l)=\bar{u}\left(e^{\prime}\right) \gamma_{\mu} u(e)$, and a hadronic current, $J^{\mu}(h)$. The hadronic current is written in the CGLN formalism as a sum over six possible gauge and Lorentz invariant amplitudes $A_{i}$ and matrices $M_{i}^{\mu}$ :

$$
\left.\mathrm{J}^{\mu}(\mathrm{h})=\overline{\mathrm{u}}\left(\mathrm{p}_{\Lambda}\right) \gamma_{5} \sum_{\mathrm{i}=1}^{6} \mathrm{~A}_{\mathrm{i}} \mathrm{M}_{\mathrm{i}}{ }^{\mu} \mathrm{u}\left(\mathrm{p}_{\mathrm{p}}\right)\right) .
$$

Expressions for the $\mathrm{M}_{\mathrm{i}}^{\mu}$ and the connection between the measured cross sections in $\mathrm{Eq}_{(2)}$ and the currents are given in Refs 5], 6], and 7].

The hadronic part of electromagnetic strangeness production is therefore reduced to the computation of the six complex amplitudes $A_{\mathrm{i}}$. In principle this should be possible starting from QCD and the quark model. In practice this has not been done, especially in the threshold region; instead models have been developed using hadronic and mesonic degrees of freedom. Figure 2 shows the diagrams which are needed. The lowest order Born terms involve proton, lambda, and kaon exchange in the $s, u$, and tchannels, respectively. Other important terms involve the exchange of the $\Sigma$, the spin-1 kaon resonances, the spin $1 / 2$ and spin $3 / 2$ nucleon resonances, and spin $1 / 2$ hyperon resonances. The coupling constants at the vertices can be extracted from the data and compared either with values predicted by $S U(3)$ or $S U(6)$, or with values obtained from other strangeness-producing reactions. For virtual photon interactions the $A_{i}$ also contain proton and kaon form factors 5].

We can consider the DESY experiment by Azemoon et al. ${ }^{3]}$, which measured e $+\mathrm{p} \rightarrow \mathrm{e}^{\prime}+\mathrm{K}^{+}+\mathrm{Y}$ for kinematics $-0.1>\mathrm{q}^{2}>-0.6 \mathrm{GeV}^{2} / \mathrm{c}^{2}$, and $1.9<\mathrm{W}<2.8 \mathrm{GeV}(\Lambda$ thieshold is at $\mathrm{W}=1.6 \mathrm{GeV})$. This experiment used two magnetic spectrometers at forward angles to detect the $\mathrm{e}^{\prime}$ and $\mathrm{K}^{+}$, with optical spark chambers, Cerenkov counters, and time-of-flight hodoscopes. Figure 3 shows the missing mass distribution $M=$

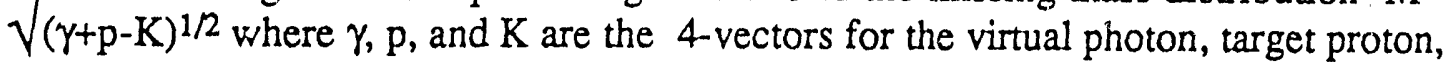
and kaon, from which it is clear that several hyperons could be isolated for study. The photon polarization varied in the fairly narrow range $0.5<\varepsilon<0.8$, and so no separation of the contribution of longitudinal and transverse virtual photons was made. Figure 4 shows the result for the transverse-transverse interference cross section and the longitudinal-transverse interference cross sections defined in Eq.(2) as a function of W, $\mathrm{q}^{2}$, and $\mathrm{t}$, compared to the sum of the longitudinal and unpolarized cross sections. One can either say that the interference terms are consistent with zero, or remark that individual points show interference cross sections roughly $10 \%$ as large as the inclusive cross sections. Without theoretical guidance one cannot extract much physics from these data, but they suggest that new experiments may have sizeable interference cross sections for which to look.

In an similar experiment done at Cornell 8 ], the $\phi$ dependence was averaged, leaving only the first two terms in Eq.(2). Figure 5 shows the $Q^{2}$ dependence of the electroproduction of $\Lambda$ and $\Sigma^{0}$ for a fixed value of the total c.m. energy W. d $\sigma / d \Omega^{*}$ is the sum of longitudinal and unpolarized transverse cross sections in the c.m. of the photon and proton. For $\Lambda$ production the cross section first rises from the real photon 


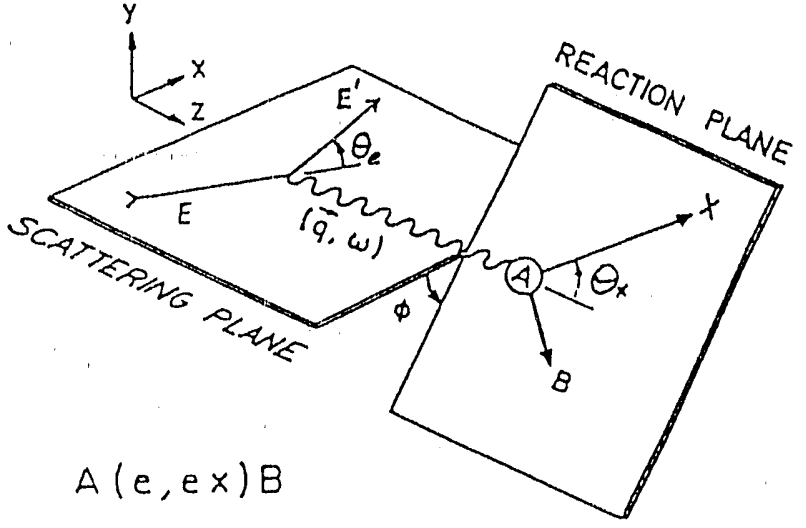

(a)

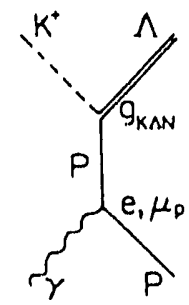

(c)

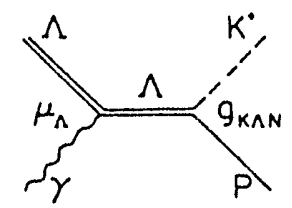

(e)

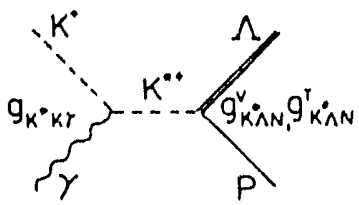

(g)

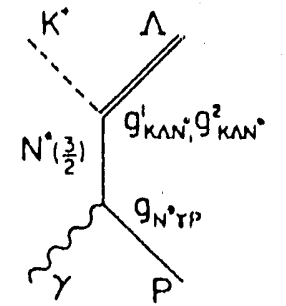

(b)<smiles>[Y]C([Y])[C@@H]([Y])[C@@H](C)F</smiles>

(d)

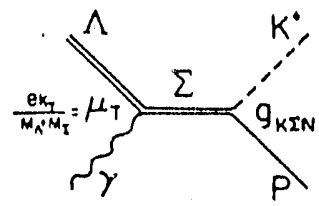

(f)

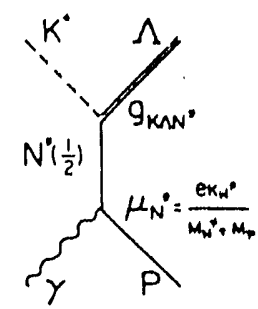

(h)

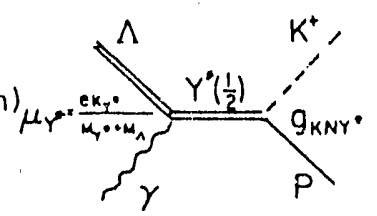

Figure 1) Kinematic variables for electroproduction. From Ref 1].
Figure 2) Diagrams for process $\gamma+\mathrm{p} \rightarrow \mathrm{K}^{+}+\Lambda$. Born terms (a-e), and resonant terms (f-h). From Ref. 15].

\section{DISCLAIMER}

This report was prepared as an account of work sponsored by an agency of the United States Governmert. Neither the United States Grovernment nor any agency thereof, nor any of their employees, makes any warranty, express or implied, or assumes any legal liability or responsibility for the accuracy, completeness, or usefulness of any information, apparatus, product, or process disclosed, or represents that its use would not infringe privately owned rights. Reference herein to any specific commercial product, process, or service by trade name, trademark, manufacturer, or otherwise does not necessarily constitute or imply its endorsement, recommendation, or favoring by the United States Government or any agency thereof. The views and opinions of authors expressed herein do not necessarily state or reflect those of the United States Government or any agency thereof. 

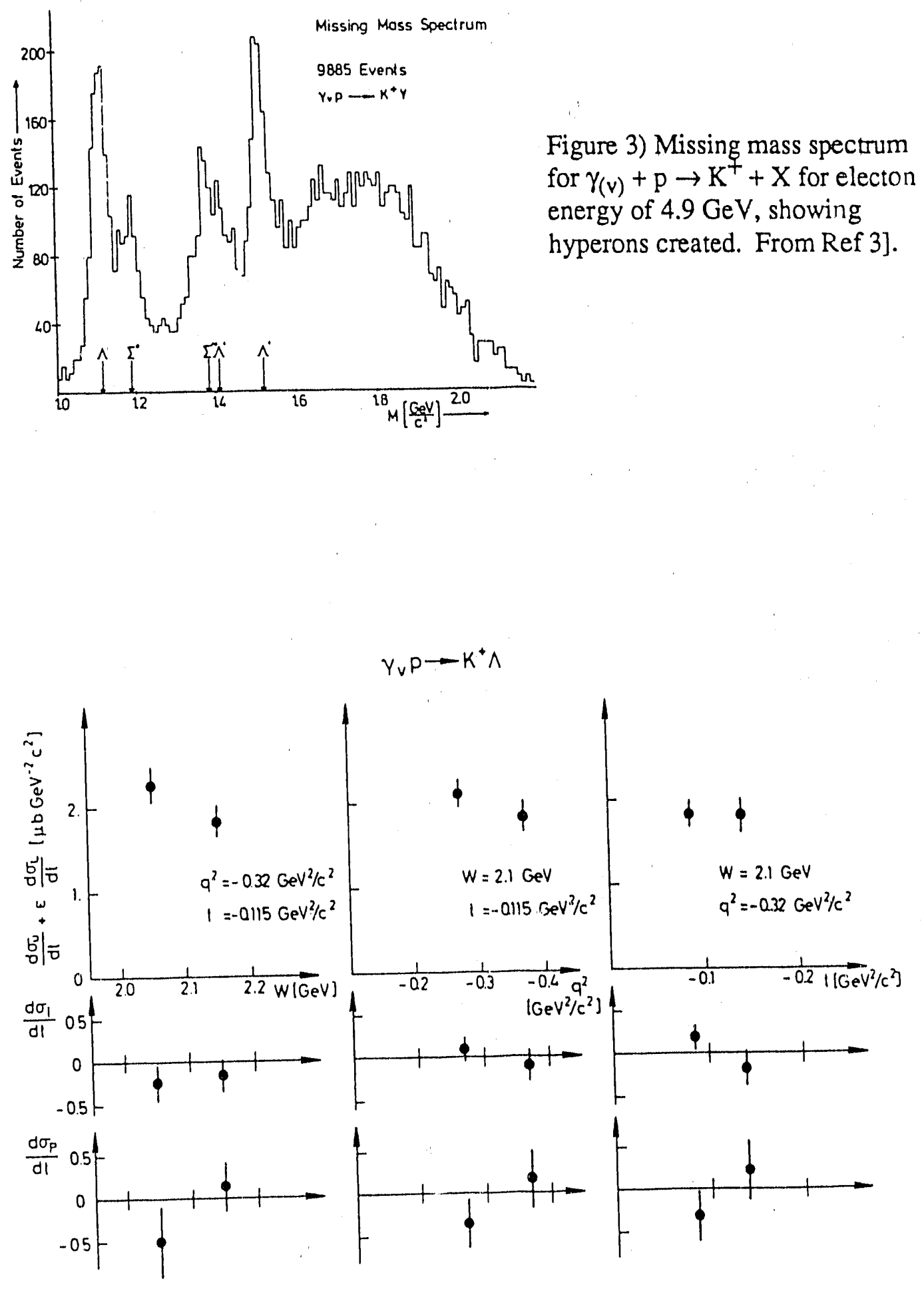

Figure 4) The cross section components in Eq. (2) for $\gamma_{(v)}+p \rightarrow K^{+}+\Lambda$ as a function of $\mathrm{W}, \mathrm{q}^{2}$, and $\mathrm{t}$ for $4.0 \mathrm{GeV}$ primary electron energy. From Ref 3$]$. 
point at $\mathrm{Q}^{2}=0$, while for $\Sigma^{0}$ production the drop is monotonic with $\mathrm{Q}^{2}$. This is interpreted as an indication that longitudinal photon contributions are important for $\Lambda$ production but not $\Sigma$ production. A rough longitudinal-transverse separation was obtained 9] by measuring at different $\varepsilon$ for the same $W$ and $Q^{2}$, supporting the conclusion that a large longitudinal component contributes to $\Lambda$ production only. A suggested explanation 3 ] is that longitudinal photons contribute strongly to kaon exchange in the t-channel, but this process is expected to be larger for $\Lambda$ production than for $\Sigma$ production since $\mathrm{g}_{\mathrm{KAN}}>\mathrm{g}_{\mathrm{K} \Sigma \mathrm{N}}$, and hence the difference in $\mathrm{Q}$ dependence.

The $Q^{2}$ dependence of $\Sigma^{0}$ production is much steeper than that for $\Lambda$ production. In quark-parton models 10],11],12] this feature was interpreted as a consequence of the decrease of the ratio $F_{1}^{\gamma n} / F_{1} \gamma p$, the deep inelastic electron-nucleon structure functions of the neutron and proton, as Bjorken $x=Q^{2} / 2 \mathrm{Mv}$ goes to 1 . In this limit the production of forward-going kaons off $u$-quarks tends to leave behind an isospin 0 pair $u-d$ quarks, from which the production of $I=1$ baryons $(\Sigma)$ is suppressed in favor of $I=0$ baryons $(\Lambda)$.

The $Q^{2}$ dependence can also be interpreted in terms of the kaon form factor. In the vector dominance picture the photon can convert to a $\phi$ meson which decays to $\mathrm{K}^{+} \mathrm{K}^{-}$before interacting with the proton. Using a vector dominance form factor, Cotanch and Hsiao 5], 13] found an rms kaon radius of 0.3 to $0.4 \mathrm{fm}$. They used a set of coupling parameters (Fig. 2) derived from early photoproduction data6]. However, an analysis by Adelseck and Wright 7] of the complete electro- and photoproduction data set gave a kaon rms radius of $0.51 \mathrm{fm}$, which agrees with more direct measurements where kaons are scattered from atomic electrons 14$]$.
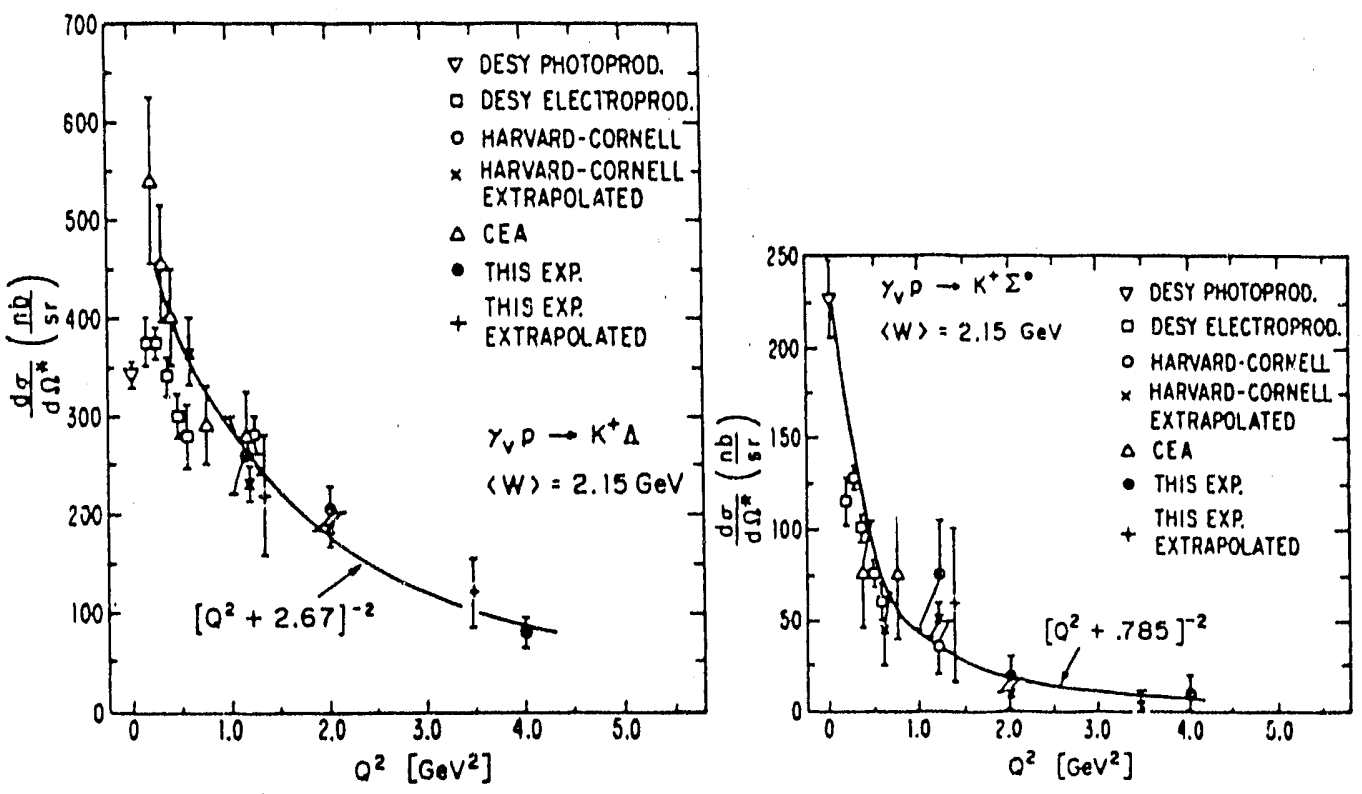

Figure 5) $\mathrm{Q}^{2}$ dependence for $\gamma_{(v)}+\mathrm{p} \rightarrow \mathrm{K}^{+}+\left\{\Lambda, \Sigma^{0}\right\}$. The trends away from the real photon point at $\mathrm{Q}^{2}=0$ differ strongly. Data are from Ref. 8] and others cited therein. 


\section{PHOTOPRODUCTION EXPERIMENTS}

In strangeness production by real photons, the longitudinal components of the cross sections vanish and the computational situation therefore simplifies somewhat. The complex invariant amplitudes are reduced from six to four in number and are given explicitly in Adelseck and Wright 7], for example.

Real photon experiments using tagged or untagged bremsstrahlung beams have been more numerous than electron scattering experiments. While being restricted to $\mathrm{q}^{2}=0$, the relative simplicity of these experiments has led to more efforts to look for polarization effects as a way to pin down to amplitudes and couplings. Figures 6 and 7 give overviews of the existing $\Lambda$ differential cross section data and polarization data 15],16],17]. The polarization data in Figure 7, which are for a kaon c.m. angle of $90^{\circ} \pm 5^{\circ}$, represent well over half of the data points ever measured. While the differential cross section is moderately well established, little more than the sign of the polarization is known.

Table I compiles the principal couplings $g_{K \Lambda N}$ and $g_{K \Sigma N}$ (see Fig. 2) which have been extracted from the data over the years. The sparseness of the data is such that a rather wide range of values for these constants has been obtained, combined with longstanding uncertainty about which resonances are really imporant in the production mechanism. The older photoproduction measurements tended to result in systematically smaller values for $\mathrm{g}_{\mathrm{KAN}}$ than the hadronically inferred values. For comparison, the table gives the SU(3) prediction of the couplings from Refs. 20] and 24], based on the experimental $\pi \mathrm{NN}$ coupling, and the values of Bozoian et al . ${ }^{21}$, who use a quarkpairing model to describe meson exchange between baryons in an MIT bag model. Also listed are some values obtained from hadronic data. Workman has emphasized the instability of the couplings against addition of many resonances on top of the Born terms 18],19], and hence the importance of trying to assess model uncertainties; his uncertainty estimates for several of the models are shown in brackets in Tabie I. Besides the values of the basic couplings, models differ in prescriptions for including resonance exchange terms to the Born amplitudes.

Recent authors have attempte? to improve the early models by exploring more combinations of resonances in their fits to data. Adelseck and Wright ${ }^{7]}$ found that including $\mathrm{K}_{1}(1280)$ exchange in the $t$-channel could increase $g_{\Lambda \mathrm{KN}}$ to the hadronic value. Tanabe, Kohno, and Bennhold ${ }^{22]}$, on the other hand, pointed out the necessity of including $\mathrm{K}^{+}+\Lambda$ final state correlations explicitly; by including a partial-wave dependent absorptive factor on top of the usual Born and resonance terms, they fit the cross section data at all energies, including beyond the typical $1.4 \mathrm{GeV}$ utoff in these studies (see Figure 8), and claim to obtain the hadronic-reaction value for $g_{K \wedge N}$. Cohen $^{23]}$ has found that taking the couplings derived from photoproduction and recalculating hadronic reactions such as low energy $\mathrm{KN}$ scattering unexpectedly produces better agreement with data than the "standard" hadronic values. He questions, however, the validity of the all diagrammatic models used to obtain elementary coupling constants from photo-kaon reactions on the grounds that the kaon mass is large on the scale of the nucleon mass, resulting in non-applicability of the Kroll-Ruderman theorem. 
Adelseck and Saghai ${ }^{24]}$ have recently re-examined all the available data on $\Lambda$ photoproduction. They find that data from just one of the dozen separate old experiments is "internally inconsistent" when compared to a simple model involving only a few exchanged particles. Removing this one data set from their analysis then leads them to a model for $\Lambda$ production which involves exchanges of only five particles: $\mathrm{K}^{*}(892), \mathrm{K} 1(1280), \Sigma^{0}, \mathrm{~N}^{*}(1440)$, and $\Lambda^{*}(1670)$; the resulting coupling constants are in agreement with the SU(3) predictions. Unfortunately, the excluded data set happens to provide most of the cross section data for kaon angles larger than $90^{\circ}$, so it may be that the simple mechanism is an artifact of a now incomplete data set 25$]$.

Numerous authors 15$], 16], 18], 22], 24]$ have pointed out that better hyperon polarization data are the necessary next step for progress in the field. Figure $7 \mathrm{a}$, for example, shows the older analysis of Renard ${ }^{16]}$ which illustrates the sensitivity of his model to $\Lambda$ polarization data: the hatched region shows the range of predictions due to reasonable variations of the couplings $g_{\Lambda K N}$ and $g_{\Sigma K N}$ (compare to Figure 6). Figure $7 \mathrm{~b}$ is from the newer analysis of Adelseck and Wright ${ }^{7]}$ (same $P$ data with minus sign), showing again the inability of present data to discriminate strongly among fits. Future experiments are expected to emphasize polarization measurements. For example, at CEBAF there are plans 26 ] to measure the $\Lambda$ polarization in detail using the toroidal spectrometer CLAS 27 .

One can itemize ${ }^{281,24]}$ sixteen observables corresponding to the combinations of photon, target, and lambda polarization that one can measure with single or double polarization measurements. Adelseck and Saghai have explored 24] the sensitivity of these observables to parameters in their model. There is sensitivity comparable to or better than that of the $\Lambda$ polarization, $P$, in several observables, essentially no data exist with which to compare. Only one non-P polarization measurement exists ${ }^{29]}$ in which the polarized target asymmetry was measured one kaon angle and three photon energies. Workman found ${ }^{30}$ ] even these few crude data points useful in comparing models.

Photoproduction data of $\Sigma^{+}$and $\Sigma^{\circ}$ (Reactions $1 \mathrm{~b}$, and 1c) are supplemental to the $\Lambda$ data, since all three reactions are described within the same framework. A useful feature of $\Sigma^{\circ}$ production is the absence of the t-channel diagrams because the photon does not couple to the $\mathrm{K}^{\circ}$. This results in a backward peak in the predicted differential cross section, which would be easy to find experimentally 31 . By detecting $\mathrm{K}^{0} \rightarrow \pi^{+}$ $\pi^{-}$this reaction will be accessible at CEBAF 26 .

Photoproduction of spin $3 / 2$ hyperons is exemplified by a Daresbury/NINA experiment ${ }^{32]}$ which examined the reaction $\gamma+\mathrm{p} \rightarrow \mathrm{K}^{+}+\Lambda(1520)$ between 2.8 and 4.8 $\mathrm{GeV} . \Lambda(1520)$ production was found to be identical to $\Lambda(1115)$ production, having the same total cross section, same s- and t- dependencies, and the same spin polarization.

\section{CONCLUSIONS}

The basic features of electromagnetic hyperon production are experimentally known and understood in terms of conventional particle production models. Theoretical progress is still possible, however, after more detailed experiments are done. Cross sections for two of the three "elementary" reactions on the proton $(\mathrm{Eq}(1))$ have 
been determined at the $10 \%$ to $20 \%$ level. But only a very crude longitudinal/transverse separation been performed for electroproduction, and no adequate measurements of the out-of-plane interference cross sections exist. Persistent difficulties with interpretation of the photoproduction data (values of coupling constants, importance of the non-Born terms) have left the picture rather incomplete. The future lies in improved coincidence measurements and the measurement of polarization observables. We can expect such progress at new facilities, such as those under cuistruction at CEBAF and Bonn.
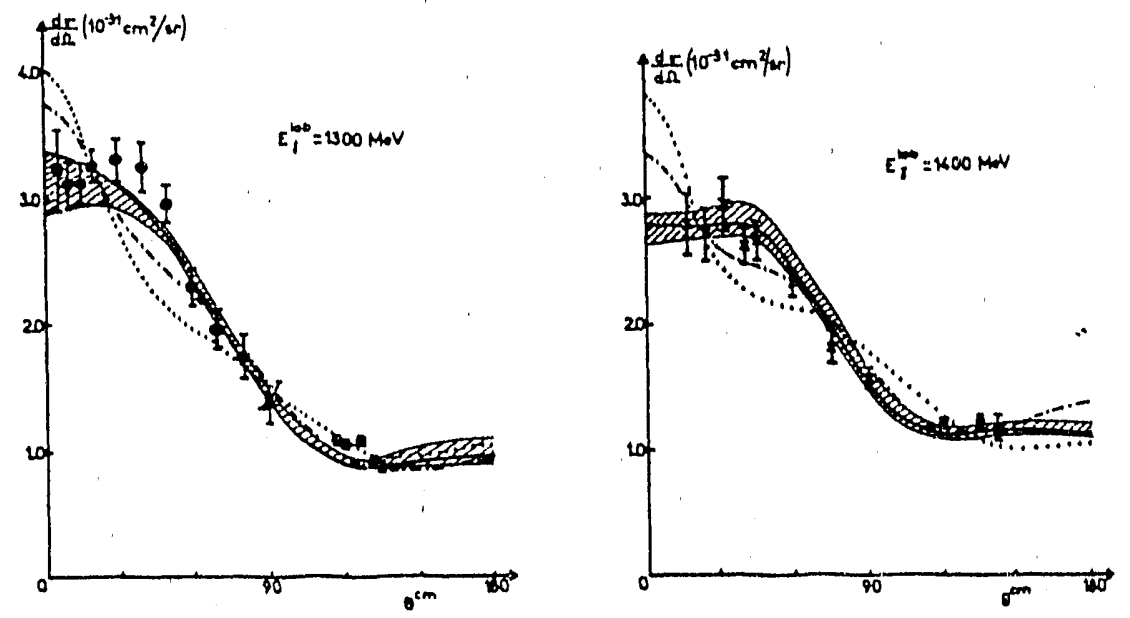

Figure 6) Differential cress sections for $\gamma+\mathrm{p} \rightarrow \mathrm{K}^{+}+\Lambda$ plotted as a function of kaon c.m. angle, from Ref 16$]$. The shaded region corresponds to $g_{\Lambda K \Lambda^{r}} / \sqrt{ } 4 \pi$ varying from 1.1 to 2.8 .

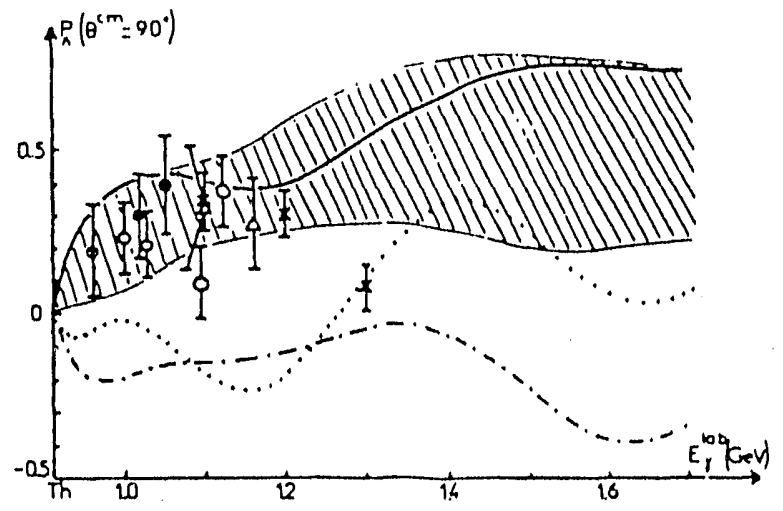

a)

Figure 7) $\Lambda$ polarization data for $\mathrm{p}\left(\gamma, \mathrm{K}^{+}\right) \Lambda$ for a

kaon c.m. angles of $90^{\circ} \pm$ $5^{\circ}$. (a) From Renard (Ref. 16]), with curves corresponding to those in Figure 6. Note the sensitivity of this model to the polarization data. (b) From by Adelseck,

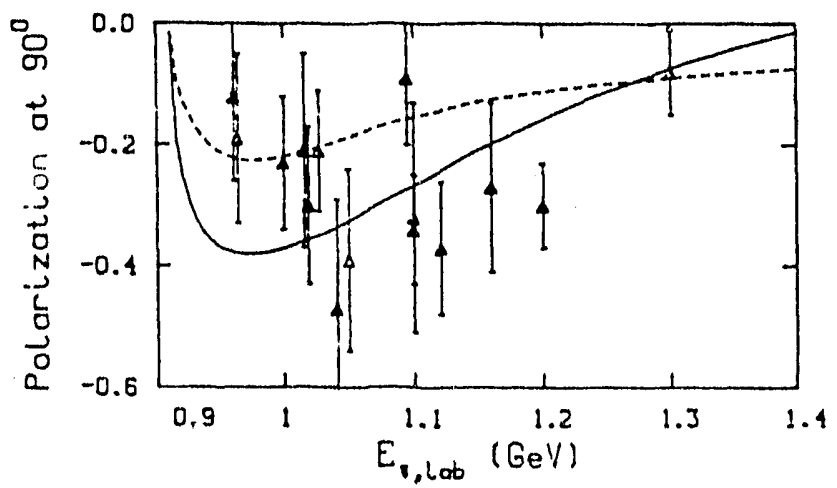

Bennhold, and Wright. (Ref 15]). Polarization has opposite sign

b) definition from (a). 


\section{Table I}

Values of $g_{K \backslash N} / \sqrt{4 \pi}$ and $g_{K \Sigma N} / \sqrt{4 \pi}$ obtained by various authors. Uncertainties in parenthesis have been computed by Workman 20] 27].

Quark model or SU(3) Prediction:

deSwart ' 63

Bozoian et al ' 83

$\frac{g_{K \Lambda N}}{\sqrt{4 \pi}} \quad \frac{g_{K \Sigma N}}{\sqrt{4 \pi}} \quad$ Reference

-3.0 to $-4.4 \quad 0.9$ to $1.3 \quad 20,24$

$-4.13+0.82 \quad 21$

From $\Lambda$ photoproduction data:

$\begin{array}{lllc}\text { Kuo '63 } & -2.0 & & 33 \\ \text { Thom '66 } & -1.1 \text { to }-2.6 & -0.9 \text { to }+1.0 & 6 \\ \text { Renard 72 } & -1.1 \text { to }-2.8 & +0.4 & 16 \\ \text { Pickering '73 } & -2.8 \text { to }-3.4 & +.9 \text { to }+1.0 & 34 \\ \text { Adelseck, Bennhold, and Wright '85 } & -1.3( \pm .1) & +2.0 & 15 \\ \text { Rosenthal } \text { et al '88 } & -0.9 & +0.6 & 35 \\ \text { Adelseck and Wright '88 } & -4.3 & -1.8 & 7 \\ \left.\quad \text { (including (e, } \text { e' }^{+}\right) \text {) } & -3.2 & -1.7 & 36 \\ \text { Cohen '89 } & -2.0 & -0.8 & 24 \\ \text { Adelseck and Saghai '90 } & -4.2( \pm 1.6) & 1.2( \pm 2.3) & \\ & & & \\ \text { photoproduction data: } & & & 37 \\ \text { Renard and Renard 71 } & -2.4 \text { to-3.6 } & +0.6 & \\ \text { Bennhold '89 } & -1.9 \text { to } 2.1 & +2.7 & 38 \\ & & & 39 \\ \text { and Y data (signs undetermined): } & & - & 40 \\ \text { Granovskii and Starikov '68 } & 2.43 & - & 41 \\ \text { Knudsen and Pietarinen '73 } & 3.5 \pm 2.5 & <1.82 & 42 \\ \text { Martin '81 } & 3.73 \pm .3 & 1.53 & \\ \text { Antolin '86 } & 3.53 & 0.9 & \\ \text { Dover and Walker '82 } & 4.62 & \end{array}$

From $\Sigma$ photoproduction data:

Renard and Renard 71

From $\mathrm{K}$ and $\mathrm{Y}$ data (signs undetermined):

Granovskii and Starikov '68

$3.5 \pm 2.5$

39

Martin '81

$3.73 \pm .3$

41

Dover and Walker ' 82

4.62

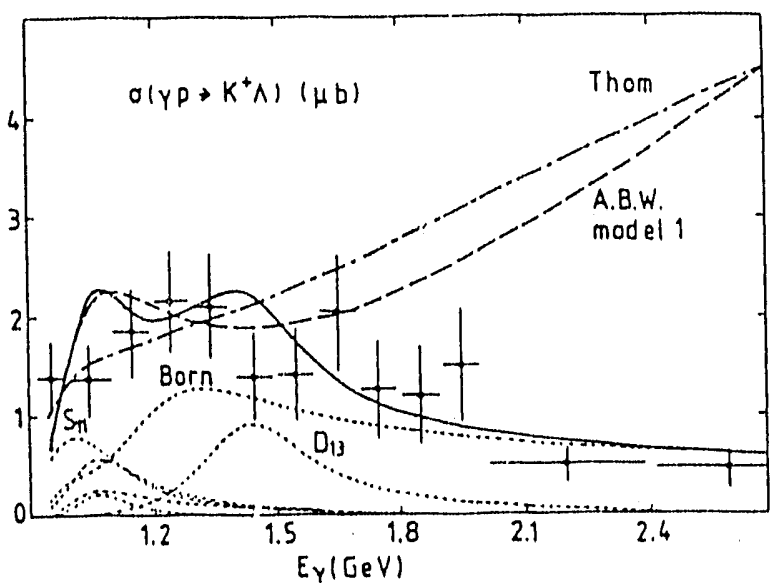

Figure 8) Total cross section. Fits are from Ref. 37] (solid and dotted lines), Ref. 6] (dot-dash), and Ref. 15] (dashed). 
1] Report of the 1987 Summer Study Group, CEBAF, VA, p. 185 (1987).

2] C.J. Bebek et al., Phys Rev Lett 32, 21 (1974).

3] T. Azemoon et al ., Nucl. Phys. B95, 77 (1975).

4] S. Boffi, C. Giusti, and F.D.Pacati, Nucl. Phys. A435, 697 (1985).

5] Stephen Cotanch and Shian Hsiao, Nucl. Phys. A450, 419c (1986).

6] H. Thom, Phys. Rev. 151, 1322 (1966).

7] R.A.Adelseck and L.E.Wright, Phys. Rev. C38 1965 (1988); Ralf Anton Adelseck, PhD thesis, Ohio University (1988).

8] C. J. Bebek et al ., Phys Rev D15, 594 (1977).

9] C. J. Bebek et al ., Phys Rev D15, 3082 (1977).

10] F.E. Close, Nucl. Phys. B73, 410 (1974).

11] O. Nachtmann, Nucl. Phys. B74, 422 (1974).

12] J. Cleymans and F.E.Close, Nucl. Phys. B85, 429 (1975).

13] Shian Hsiao and Stephen Cotanch, Phys. Lett. 28, 200 (1985).

14] E.B.Dally et al.., Fhys Rev Lett 45, 232 (1980).

15]A vailable data are summarized in R.A.Adelseck, C. Bennhold, and L.E.Wright, Phys. Rev. C32, 1681 (1985).

16] Y.Renard Nucl. Phys. B40, 499 (1972); F. M. Renard and Y. Renard, Nucl. Phys. B 25, 491 (1971).

17] T. Fujii et al.., Phys. Rev. D 2,439 (1970).

18] R.L.Workman, Phys Rev C39 , 2076 (1989); reply to comment by Workman in R.A.Adelseck and L.E.Wright, Phys Rev C39 , 2078 (1989).

19] R.L. Workman, Proc. of "Few Body XII", Vancouver, ed. B.K.Jennings (1989).

20] J.J.deSwart, Rev. Mod. Phys. 35, 916 (1963).

21] M. Bozoian, J.C.H. van Doremalen, and H.J.Weber, Phys. Lett 122B, 637 (1983).

22] H. Tanabe, M. Kohno, and C. Bennhold, Phys Rev C39, 711 (1989).

23] Joseph Cohen, Phys. Lett. B 192291 (1987); Joseph Cohen, Phys. Rev. C 37 , 187 (1988).

24] R.A.Adelseck and B. Saghai, Phys. Rev. C42, 108 (1990).

25] R. Workman, preprint and private communication.

26] CEBAF proposal 89-04, Camegie Mellon, Catholic U., V.P.I., CEBAF, Florida State, Los Alamos; R. Schumacher, Spokesman (1990); CEBAF proposal 89-43, CEBAF N* Collaboration, L.Dennis and H. Funsten, Spoeksmen (1990).

27] CEBAF Conceptual Design Report, April, (1990).

28] I.S.Barker, A. Donnachie, and J.K..Storrow, Nucl. Phys. B95, 347 (1975).

29] K.H.Althoff $t$ al., Nucl. Phys. B137, 269 (1978).

30] R.L Workman, Phys. Rev. C42, 781 (1990).

31] C. Bennhold, private communicatiori.

32] D.P.Barber et al., Z. Physik C7, 17 (1980).

33] T.K.Kuo, Phys. Rev. 129, 2264 (1963).

34] A. R. Pickering, Nucl. Phys. B66, 493 (1973).

35] A. S. Rosenthal, Dean Halderson, Kimberly Hodgkinson, and Frank Tabakin, Annals of Physics 184, 33 (1988).

36] Joseph Cohen, Phys. Rev. C39, 2285 (1989).

37] C. Bennhold, Phys. Rev. C39, 1944 (1989).

38] Ya. I. Granovskii and V.N. Starikov, Sov. J. Nucl. Phys. 6, 444 (1968).

39] C.P.Knudsen and E. Pietarinen, Nucl. Phys. B57, 637 (1973).

40] A.D.Martin, Nucl. Phys. B179, 33 (1981).

41] J. Antolin Z. Physik C31, 417 (1986).

42] Carl B. Dover and George E. Walker, Phys. Rep. 89, 1 (1982). 

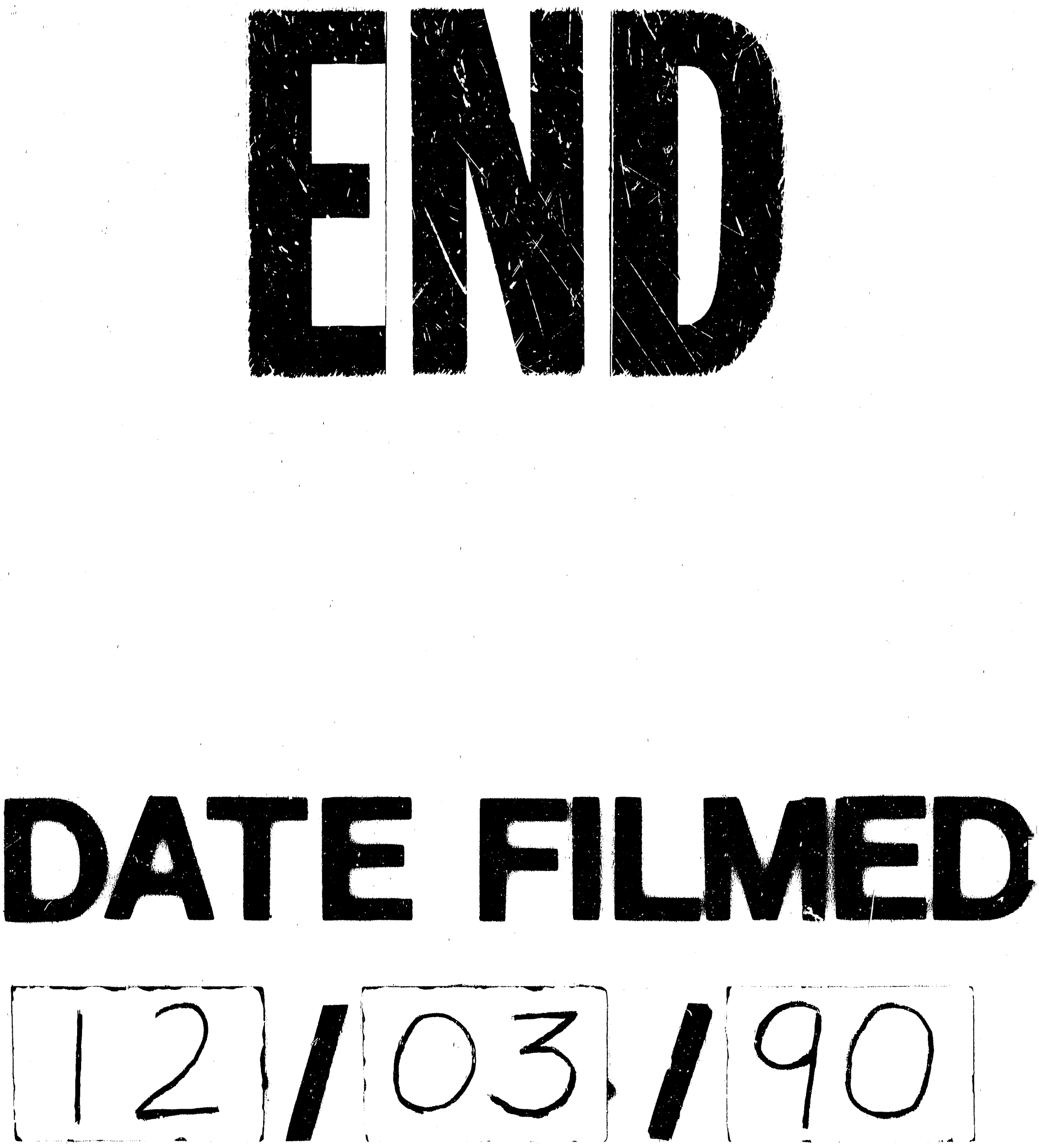